\title{
Health Literacy and Associated Factors Among Hmong American Immigrants: Addressing the Health Disparities
}

\author{
Belle P. Khuu' ${ }^{1}$ · Hee Y. Lee ${ }^{1}$ Anne Q. Zhou ${ }^{2}$
}

(C) Springer Science+Business Media New York 2017

\begin{abstract}
Hmong Americans face a disproportionate health burden ranging from the high prevalence of diabetes to depressive disorders. Little research attention has been paid toward exploring contributing factors to this disparity. As such, the present study seeks to fill the gap in the literature by examining the health literacy levels in Hmong Americans and its associated factors. The present study employed Andersen's behavioral model of health service as the theoretical framework. A cross-sectional survey research design was used and information was gathered from 168 Hmong American immigrants. Participants were recruited using a purposive sampling strategy. A multiple regression analysis was conducted to identify the factors linked to health literacy. Approximately half of the participants had low health literacy and reported that they did not understand health information well. Health literacy levels were found to differ significantly based on the number of years participants have lived in the U.S., their social or religious group attendance, health status, and whether they had difficulties with activities of daily living. Our exploratory findings could be used prompt more research to help inform the development of interventions aiming to improve health literacy levels and address the health disparities in Hmong American Population.
\end{abstract}

Belle P. Khuu

bkhuu@umn.edu

1 School of Social Work, University of Minnesota, Twin Cities, MN, USA

2 Department of Psychology, University of Minnesota, Twin Cities, MN, USA
Keywords Hmong Americans $\cdot$ Health literacy $\cdot$ Asian Americans $\cdot$ Immigrants $\cdot$ Health disparities $\cdot$ Andersen behavioural model

\section{Introduction}

Hmong American immigrants are among the fastest growing ethnic groups in the United States (U.S.), with an increase of $40 \%$ from 2000 to 2010 [1]. However, they remain a critically underserved and under researched immigrant group within the Asian American community. This is concerning given the disproportionate number of health risks faced by the Hmong American community, such as the high prevalence of chronic conditions especially hepatitis B and cancer [2-5], as well as mental health concerns $[6,7]$. Several contributing factors to the health disparities among Hmong American immigrants are their low use of preventive care, high rejection of formal health services, and delayed help seeking of critical health services [8]. For example, Yang et al. [9] found that roughly $16 \%$ of Hmong American immigrant women reported ever having a mammogram compared to $82 \%$ for non-Hispanic white women.

Another potential contributing factor for the health disparity issue could be that of low health literacy. Health literacy is often defined as "the degree to which individuals have the capacity to obtain, process and understand basic health information and services needed to make appropriate health decisions" [10, p. vi], and has been demonstrated to be an important factor that impacts health outcomes. Although health literacy has not yet been extensively studied among Hmong American immigrants, a small number of studies suggest that low health literacy might be a problem among this population [11]. Hmong American immigrants have reported views of U.S. health care services as 
confusing and incompatible with their beliefs about health [12], suggesting an overall distrust of the Western health care system. This distrust can lead to increased delay of seeking critical health services [13] and increased likelihood of developing chronic and serious illnesses [14]. Additionally, in one of few a studies on health literacy and Hmong Americans, Lee and Vang [13] found that Hmong Americans reported low overall cancer literacy and cancer prevention knowledge. However, the potentially low health literacy within Hmong American immigrants is not just in regards to physical health issues but also mental health concerns. In a study that examined mental health literacy in Hmong and Cambodian American elders, Lee et al. [15] found that participants lacked basic knowledge about mental disorders and available mental health services, and that the Hmong language itself did not have terminologies for major mental disorders.

Yet, despite the evidence that health literacy is significantly connected to health disparities and utilization of services, and that health literacy is potentially problematic in this population, a scant number of studies over the years have explored health literacy among Hmong Americans. However, health literacy may be important factor towards addressing the immense health disparities and low utilization of formal health services among Hmong American immigrants. Thus, it is important and timely to closely examine health literacy within this population.

\section{Current Study}

The purpose of this study is to examine the health literacy status of Hmong American immigrants as well as identify significant factors associated with health literacy within this population. It seeks to fill the existing gaps in the literature regarding the link between health literacy among Hmong Americans. The relevant factors explored in the present study were chosen based on Andersen's behavioral model of health services use [16]. While the original intent of the model was to examine malleable individual factors related to health care services utilization, this model has also been found to be a good framework for exploring health literacy in ethnic and racial minority groups $[17,18]$. The model categorizes factors into predisposing characteristics, enabling resources, and need based factors [16]. Predisposing characteristics are defined as exogenous factors that facilitate or hinder the use of services, while enabling resources are defined as important conditions that would aid individuals in seeking health services. Finally, need factors are factors that measure the perceived need for health services.

Using this model, our conceptual framework explores the factors that significantly related to health literacy in Hmong Americans by adopting the three main components: predisposing factors (age, years in the U.S., and marital status), enabling factors (income, usual place of care, and social or religious attendance), and need factors (health status and activities of daily living [ADLs]). The choice to include usual place of medical care in the model instead of health insurance status was largely due to extant literature indicating a high rate of health insurance among refugees and documented immigrants [19]. Additionally, social or religious group attendance was included to explore the effects of these variables on health literacy [20]. Specifically religion was included due to the finding that religion is an integral feature in the daily lives of many Hmong Americans, specifically the Shamanism and Christianity sectors [21]. To our knowledge, this study is the first to examine health literacy and predictive factors of health literacy among Hmong Americans.

\section{Method}

\section{Research Design and Data Collection}

This study employed a cross-sectional survey research design. Study participants were recruited through a purposive sampling frame through posting flyers at ethnic grocery markets or beauty salons, word of mouth, and social media. The eligibility criteria were self-identifying as a Hmong American, being aged 18 and over, and willingness and ability to consent to participate in the study. Informed consent was obtained from all individual participants included in the study. For participating in the study, participants were offered the option to be entered into a raffle to win one of two $\$ 50$ gift cards. Responses to a questionnaire were obtained through in-person interviews or online surveys with the in-person interviews conducted by three trained, bilingual Hmong American research assistants. Interviews were conducted in Hmong or English, as determined by each participant's preference. In addition to in-person interviews, the survey was administered via Survey Monkey, with the online survey also offered in either the Hmong or English language. This study was reviewed and approved by the University of Minnesota Institutional Review Board.

\section{Measures}

\section{Dependent Variables}

Due to the absence of culturally and linguistically appropriate health literacy instruments developed for Hmong Americans, this study measured health literacy using two questions, "I am confident to understand health information given by health care professionals." and "I have experiences 
of missing medication because I did not know how to take medication." These questions were designed to tap into a person's understanding of health information leading to a tangible health outcome (i.e., missing medication). Although these questions are not a comprehensive measure of health literacy, they were meant to serve as a proxy for the core concepts within health literacy. These questions were among a set of questions about the participants health beliefs, attitudes, and behaviors. The questions, along with the other ones, were piloted with a group of Hmong American immigrants in the community. Possible responses were measured on a 5-point Likert-type scale (strongly disagree [1] to strongly agree [5]) with higher score indicating a higher level of health literacy.

\section{Independent Variables}

The present study chose three sets of independent variables as guided by Andersen's behavioral model. For the first set, predisposing factors of years in the U.S., marital status, and age were explored. Number of years in the U.S. was entered as a continuous variable while age and marital status were entered as dichotomous variables. Due to the unequal distribution of age in our sample, age was dichotomized into 18-30 and 31 and greater. Marital status was coded as "married" versus "non-married," with those who were single, divorced, and widowed included in the non-married category. Next, enabling factors such as household monthly income (categorized into $\$ 1999$ or less, $\$ 2000-\$ 5999$, and $\$ 6000$ or more), usual place of medical care was dichotomized as yes/no, and attendance in social or religious groups was dichotomized as yes/no. Finally, need factors included health status (rated as either "fair to poor health" or "good to excellent") and ADLs (dichotomized as having ADL problems [yes] or none [no]).

\section{Data Analysis}

Univariate and bivariate analyses were conducted to determine the demographic characteristics of the study sample and their health literacy status. T-tests were conducted to compare the reported health literacy scores based relevant dichotomized demographic characteristics. For the income variable, ANOVA was performed to determine whether there were any differences in health literacy among the three income groups. For the age variable, a Pearson's correlation was computed to examine the correlation between age and health literacy. Additionally, to explore the main research question regarding the predictive factors of health literacy based on Andersen's theoretical framework, a hierarchical regression analysis was used. All of the statistical procedures were performed using the SPSS 22.0 software package (IBM Corp., 2013).

\section{Results}

\section{Sociodemographics of the Sample}

One hundred sixty-eight Hmong Americans were participated in this study. Briefly, there were more females (70\%) than males and more than half $(64 \%)$ of the study participants were 30 years old or younger $(M=29.2, S D=8.6)$. Additionally, participants in the present study reported that they had been in the U.S. for a relatively long duration (mean $=25.6$ years, $S D=6.2$ ). Approximately $41 \%$ $(n=69)$ reported currently being married or cohabitating, and roughly half of participants held a bachelor's degree or higher $(45 \%)$. The majority of the sample reported being employed $(79 \%, \mathrm{n}=131)$ with $37 \%$ of participants reporting a monthly income between $\$ 2000$ and $\$ 5999$. A majority of the sample $(70 \%)$ reported having a usual place of medical care and $87 \%$ stated that they had health insurance. Roughly $64 \%$ of respondents did not attend a social club or religious group and a little less than half of the study sample reported their health as being "very poor" to "fair" (45\%). About $10 \%$ of the study sample reported current difficulties in activities associated with ADLs. The study sample's complete sociodemographic characteristics with association to health literacy are shown in Table 1.

\section{Health Literacy Status}

More than half of the participants (56.5\%) reported that they "strongly disagree" to "somewhat disagree" when asked whether they understood health information well. Additionally, $18.3 \%$ of the sample stated that they "strongly agree" to "somewhat agree" when asked if they've ever missed taking medication because they did not know how to take the medication. Overall health literacy was also examined by taking the summation of each participant's response, with possible scores ranging from 2 to 10 with mean score of $6.9(S D=2.8)$ (Table 2).

\section{Factors Predicting Health Literacy}

Table 3 presents the hierarchical results on predictors of health literacy guided by Andersen's behavioral model. In Step 1, predisposing factors were found to explain 6\% of the variance in health literacy. Both age and the number of years in the U.S. were significant related to health literacy $(p<.05, p \leq .01)$. In Step 2, after adding income, usual place of care, and social or religious group attendance, the enabling factors were found to explain $12 \%$ of variance. Age and years in the U.S. remained statistically significant, and social or religious group attendance $(p<.05)$ was also found to be significantly related to health literacy. In Step 3, the addition of the need 
Table 1 Sociodemographic of the participants

\begin{tabular}{|c|c|c|c|c|}
\hline & \multicolumn{4}{|c|}{ Frequency health literacy } \\
\hline & $\%(\mathrm{~N})$ & $\mathrm{M}(\mathrm{SD})$ & $\mathrm{T} / \mathrm{F}$ & $p$ \\
\hline Gender & & & 1.73 & 0.09 \\
\hline Male & $29.8(50)$ & $7.38(1.31)$ & & \\
\hline Female & $70.2(118)$ & $7.76(1.60)$ & & \\
\hline Age $($ mean $=29.24, S D=8.60)$ & & & -0.24 & 0.81 \\
\hline $18-30$ & $64.3(108)$ & $5.07(1.41)$ & & \\
\hline$\geq 31$ & $35.7(60)$ & $5.13(1.70)$ & & \\
\hline Education & & & 3.48 & 0 \\
\hline$<$ Bachelor's degree & $45.4(74)$ & $7.23(1.49)$ & & \\
\hline$\geq$ Bachelor's degree & $54.6(89)$ & $8.02(1.41)$ & & \\
\hline Marital status & & & -0.15 & 0.88 \\
\hline Single or other & $59.2(100)$ & $7.66(1.41)$ & & \\
\hline Married or has partner & $40.8(69)$ & $7.62(1.68)$ & & \\
\hline Employment & & & 1.78 & 0.08 \\
\hline No & $20.6(35)$ & $7.26(1.36)$ & & \\
\hline Yes & $78.9(131)$ & $7.76(1.55)$ & & \\
\hline Income & & & 0.91 & 0.4 \\
\hline$\$ 1999$ or less & $32.0(54)$ & $7.48(1.38)$ & & \\
\hline$\$ 2000-\$ 5999$ & $36.7(62)$ & $7.60(1.59)$ & & \\
\hline$\$ 6000$ or more & $31.4(53)$ & $7.87(1.52)$ & & \\
\hline Usual place of care & & & 2.26 & 0.03 \\
\hline No & $29.8(50)$ & $7.24(1.36)$ & & \\
\hline Yes & $70.2(118)$ & $7.81(1.56)$ & & \\
\hline Social or religious group attendance & & & 2.87 & 0.01 \\
\hline No & $63.7(107)$ & $7.38(1.59)$ & & \\
\hline Yes & $36.3(61)$ & $8.07(1.28)$ & & \\
\hline Health status & & & 4.49 & 0 \\
\hline Very poor to fair & $45.2(76)$ & $7.09(1.49)$ & & \\
\hline Good to excellent & $54.8(92)$ & $8.10(1.41)$ & & \\
\hline Health insurance & & & 1.23 & 0.22 \\
\hline No & $13.0(22)$ & $7.27(1.64)$ & & \\
\hline Yes & $87.0(147)$ & $7.70(1.50)$ & & \\
\hline Difficulties with ADLs & & & -2.39 & 0.02 \\
\hline No & $89.9(151)$ & $7.74(1.50)$ & & \\
\hline Yes & 10.1(17) & $6.82(1.51)$ & & \\
\hline & & Mean $(S D)$ & $r$ & Sig \\
\hline Years in USA & & $25.61(6.15)$ & 0.186 & 0.02 \\
\hline Health literacy & & $7.65(1.52)$ & - & - \\
\hline
\end{tabular}

Numbers can vary due to missing values and might not add up to $100 \%$ due to rounding

factors to the model further explained another $9 \%$ of the variance. However, after Step 3, age was no longer significantly related to health literacy. Years in the U.S. and social or religious group attendance continued to be statistically significant $(p<.05)$. In addition, the need factors of health status as well as difficulties with ADLs were also found to be significantly related to health literacy $(p \leq .001, p<.05)$. The final model with all variables was found to explain $22 \%$ of the variance in health literacy $(p<.001)$.

\section{Discussion}

The present study provided quantitative evidence that suggested poor health literacy in the Hmong American 
Table 2 Descriptive analysis on health literacy

\begin{tabular}{llllll}
\hline & $\mathrm{N}(\%)$ & & \multicolumn{1}{c}{ Total $M(S D)$} \\
\cline { 2 - 6 } & Strongly disagree & Disagree & Somewhat disagree/agree & Agree & Strongly agree \\
\hline $\begin{array}{l}\text { I am confident to understand health } \\
\text { information given by health care profes- } \\
\text { sionals }\end{array}$ & $15(8.9)$ & $9(5.3)$ & $70(41.4)$ & $48(28.4)$ & $27(16.0)$ \\
\hline & $\mathrm{N}(\%)$ & & & $3.37(1.09)$ \\
\cline { 2 - 6 } & Strongly agree & Agree & Somewhat disagree/agree & Disagree & Strongly disagree \\
\hline $\begin{array}{l}\text { I have an experience of missing medica- } \\
\text { tion because I did not know how to take } \\
\text { the medication }\end{array}$ & $7(4.1)$ & $5(3.0)$ & $19(11.2)$ & $42(24.9)$ & $96(56.8)$ \\
\hline
\end{tabular}

The second item was reverse scored for regression analysis

Table 3 Hierarchical regression analysis on health literacy $(\mathrm{N}=160)$

\begin{tabular}{|c|c|c|c|c|c|c|c|c|c|}
\hline & \multicolumn{3}{|c|}{ Model 1} & \multicolumn{3}{|c|}{ Model 2} & \multicolumn{3}{|l|}{ Model 3} \\
\hline & $B$ & $S E$ & $\beta$ & $B$ & $S E$ & $\beta$ & $B$ & $S E$ & $\beta$ \\
\hline \multicolumn{10}{|l|}{ Predisposition factors } \\
\hline Age & -0.65 & 0.32 & $-0.20 *$ & -0.66 & 0.31 & $-0.21 *$ & -0.51 & 0.30 & -0.16 \\
\hline Years in the US & 0.07 & 0.02 & $0.30 * *$ & 0.06 & 0.02 & $0.24 *$ & 0.05 & 0.02 & $0.22 *$ \\
\hline Marital status & 0.02 & 0.28 & 0.01 & -0.02 & 0.28 & -0.01 & 0.08 & 0.27 & 0.03 \\
\hline \multicolumn{10}{|l|}{ Enabling factors } \\
\hline Income & & & & 0.09 & 0.16 & 0.05 & 0.04 & 0.15 & 0.02 \\
\hline Usual place of care & & & & 0.42 & 0.27 & 0.13 & 0.23 & 0.26 & 0.07 \\
\hline Social or religious attendance & & & & 0.56 & 0.25 & $0.18 *$ & 0.58 & 0.24 & $0.18^{*}$ \\
\hline \multicolumn{10}{|l|}{ Need factors } \\
\hline Health status & & & & & & & 0.80 & 0.23 & $0.26 * * *$ \\
\hline ADLs difficulties & & & & & & & -0.80 & 0.38 & $0.16^{*}$ \\
\hline$F$ & $3.58^{*}$ & & & $3.60 *$ & & & $5.27 * * *$ & & \\
\hline$R^{2}$ & $0.06^{*}$ & & & $0.12 *$ & & & $0.22 * * *$ & & \\
\hline$\Delta R^{2}$ & $0.05 *$ & & & $0.09 *$ & & & $0.17 * * *$ & & \\
\hline
\end{tabular}

population. About half of the participants reported that they did not understand health information well. Additionally, a fifth of the study sample reported having previously experienced difficulties with functional aspect of health literacy, such as medication management. This suggests that the overall health literacy level found in this sample may be lower in comparison to the general population where the prevalence of marginal health literacy ranges from 20 to $26 \%$ [22]. Although a few sporadic studies that have suggested that low health literacy is a problem in the Hmong American immigrant population [12], the present study is the first to demonstrate such by using a measure of health literacy. This finding is concerning given that health information is necessary for success in navigation of the health care system. Specifically, functional literacy is critical for the management of preventive health behaviors and treatments and is the basic skill needed to effectively navigate the demands of daily living [23]. The results suggest the need to not only focus on improving health knowledge, but also the need to prioritize the assessment and intervention of functional aspect of health literacy among Hmong American immigrant population.

The hierarchical regression analyses found that only one predisposing factor (i.e., years in the U.S.), one enabling factor (i.e., social or religious attendance), and both need factors (i.e., health status and ADLs difficulties) were significant in predicting health literacy levels among Hmong American immigrants. The findings regarding living years in the U.S. and its positive relationship with health literacy are in line with previously published studies (Garbers et al. 2010). The present study's finding regarding the significant relationship between years in the U.S. with health literacy suggests that more attention should be paid to acculturation factors in general. While limited English proficiency (LEP) has previously been found to be an important barrier to health literacy, particularly for various Asian American 
subgroups [17, 24], acculturation is dynamic and complex process and has been found to play a significant role in the various aspects of health literacy [25]. As such, more attention needs to be paid to the exploring the different aspects of acculturation.

Interestingly, age was not significant after controlling for all other variables, contrasting with previous studies [24-28]. Previous research have suggested that the elderly, often defined as age 65 and older, tended to report lower health literacy than their younger counterparts. Although age was initially significantly related to health literacy, our findings ceased to replicate the association between age and health literacy once other variables were introduced. This finding highlights the importance of taking a more comprehensive approach to studying factors related to health literacy.

Among the enabling factors, attendance in various social and religious groups was also found to be significantly related to health literacy. While the relationship between religion and health has previously been studied [27-32], less is known about its role in health literacy. However, there is research that generally support social support as a mediating or moderating factor of health literacy. For example, Lee et al. [33] found that social support may provide some buffering for those with low health literacy. Osborn et al. [34] also found that social support plays a major role in the pathway linking health literacy to diabetes self-care. Similarly, Johnson et al. [20] demonstrated that among those with limited health literacy having a trusted confidant, a subtype of social support, increased their level of medical information adherence. This an area that future researchers may want to explore further so that greater understanding regarding the significant relationship can be gained, particularly given that religion and religious practices are considered important influences [35].

Similarly, the finding that both health status and ADLs difficulties were identified to be significantly related to health literacy also mirrored results from previous studies Wolf et al. [28], Kindig et al. [36]. Those with limited health literacy had worse health status, were less likely to be able to manage their health, and had lower use of preventive services. As need factors associated independently with health literacy, these may be important factors that future interventions could target.

\section{Limitations of the study}

There are several limitations to this study. First, as mentioned above, the study employed a non-parametric sampling technique that limits the generalizability of the findings to the entire Hmong American population. Additionally, the current sample's demographics are slightly different from the Hmong American population at large with a higher proportion of female, slightly older, more educated, and higher income individuals [37, 38], again affecting the generalizability of the results to the Hmong American population. Second, the use of two different modes of survey administration (i.e., online and in-person interviews) posed limitations on the interpretation of the study's findings. Third, though the study initially aimed for an equal number of male and female participants, there were more females in the study (70\%), limiting the scope of our gender analysis. Lastly, due to the lack of linguistically and culturally matched health literacy measurements, this study used a two-item questionnaire that likely did not tap into all health literacy domains [39].

\section{Implications of the Findings for Practice and Policy}

The present exploratory study of health literacy in Hmong American immigrants has the potential for several significant implications. The findings highlight the importance of studying this population, not as a part of a larger aggregated Asian American group, but as its own heterogeneously distinct ethnic group as well as the importance of studying cultural and contextual factors. It is imperative that more population based and longitudinal health literacy studies are conducted among Hmong Americans. Further research could confirm the study's finding enabling for causal claims. This strategy can help inform the development of strategic plans to address and allocate health resources for those at an elevated risk of not obtaining or understanding basic health information. In particular, individuals who are newly settled in the U.S., at increased risks for health conditions, or those who are socially or religiously disconnected may be important groups to target. However, it would be important for health information to not just be linguistically matched but also be presented in ways that incorporate the patient's existing cultural understanding of health and illness. For example, instead of a language concordant brochure that provides textual information, alternative modes of health messaging to improve health literacy can be delivered through oral storytelling, visual images, or digital narratives that center on patients' existing cultural health views.

Additionally, our preliminary finding on the significance of social and religious attendance has tremendous implications for assessment and clinical practices, particularly given the role social or religious support systems play in the Hmong American community [21]. Clinicians may benefit from taking on a more strengths-based approach by exploring social and religious participation as a part of assessment and intervention, rather than a deficit approach by just focusing on low health literacy levels. Knowing the patient's level and type of social and religious connection can potentially enable the health provider to capitalize on 
the patient's built-in resources, such as existing social ties, thereby increasing the patient's health literacy level. In sum, the health disparities among Hmong Americans and other immigrant populations can be ameliorated through more culturally and empirically targeted health literacy research and intervention approaches.

Acknowledgements The research team would like to express their gratitude to the Hmong American immigrants who participated in this study and dedicate this research to the memory of Mr. Andrew Park.

Funding This study was supported by research fund of the Ucare Foundation, MN.

\section{Compliance with Ethical Standards}

Conflict of interest The authors declare that they have no conflict of interest.

\section{References}

1. Asian American Center for Advancing Justice. (2015). A Comunity of constrasts: Asian Americans, Native Hawaiians and Pacific Islanders in California, 2015. Retrieved from: http:// www.advancingjustice.org/publication/community-contrastsdemographic-report-asian-americans-native-hawaiians-andpacific.

2. Ross, J. A., Xie, Y., Kiffmeyer, W. R., Bushhouse, S., \& Robison, L. L. (2003). Cancer in the Minnesota Hmong population. Cancer, 97(12), 3076-3079. doi:10.1002/cncr.11443.

3. Sheikh, M. Y., Atla, P. R., Raoufi, R., Sadiq, H., \& Sadler, P. C. (2012). Prevalence of hepatitis B infection among young and unsuspecting hmong blood donors in the Central California Valley. Journal of Community Health, 37(1), 181-185. doi:10.1007/ s10900-011-9434-y.

4. Yang, R. C., Mills, P. K., \& Riordan, D. G. (2004). Cervical cancer among Hmong women in California, 1988 to 2000. American journal of preventive medicine, 27(2), 132-138.

5. Mills, P. K., Yang, R., \& Riordan, D. (2005). Lymphohematopoietic cancers in the united farm workers of America (UFW), 1988-2001. Cancer Causes \& Control, 16(7), 823-830

6. Lee, S., \& Chang, J. (2012). Mental health status of the Hmong Americans in 2011: Three decades revisited. Journal of Social Work in Disability and Rehabilitation, 11(1), 55-70. doi:10.1080 /1536710X.2012.648117.

7. Portis, A. J., Laliberte, M., Tatman, P., Moua, M., Culhane-Pera, K., Maalouf, N. M., \& Sakhaee, K. (2010). High prevalence of gouty arthritis among the Hmong population in Minnesota. Arthritis Care and Research, 62(10), 1386-1391. doi:10.1002/ acr.20232.

8. Sparks, S. M. A. (2014). Considerations of culture and community in the production of Hmong health. Family and Consumer Sciences Research Journal, 42(3), 224-234. doi:10.1111/ fcsr.12057.

9. Yang, R. C., Mills, P. K., \& Dodge, J. L. (2006). Cancer screening, reproductive history, socioeconomic status, and anticipated cancer-related behavior among Hmong adults. Asian pacific Journal of Cancer Prevention, 7(1), 79.

10. Ratzan, S. C., \& Parker, R. M. (2000). National Library of Medicine Current Bibliographies in Medicine: Health Literacy. NLM Pub. No. CBM 2000-1.
11. Kong, B. H. (1999). Cancer Beliefs of the Hmong. University of Wisconsin-Milwauke.

12. Gensheimer, L. (2006). Learning from the experiences of hmong mental health providers. Hmong Studies Journal, 7, 1-31.

13. Lee, H. Y., \& Vang, S. (2010). Barriers to cancer screening in Hmong Americans: The influence of health care accessibility, culture, and cancer literacy. Journal of Community Health, 35(3), 302-314. doi:10.1007/s10900-010-9228-7.

14. Reznik, V., Cooper, T., MacDonald, D., Benador, N., \& Lemire, J. (2001). Hais Cuaj Txub Kaum Txub-to Speak of All things: A Hmong Cross-Cultural Case Study. Journal of Immigrant Health, 3(1), 23-30. doi:10.1023/A:1026658518002.

15. Lee, H. Y., Lytle, K., Yang, P. N., \& Lum, T. (2010). Mental health literacy in Hmong and Cambodian elderly refugees: A barrier to understanding, recognizing, and responding to depression. The International Journal of Aging and Human Development, 71(4), 323-344. doi:10.2190/AG.71.4.d.

16. Anderson, R. M. (1995). Revisiting the behavioral model and access to medical care: Does it matter?" Journal of Health and Social Behavior, 36(1), 1-10.

17. Lee, H. Y., Rhee, T. G., Kim, N. K., \& Ahluwalia, J. S. (2015). Health literacy as a social determinant of health in Asian American immigrants: Findings from a population-based survey in California. Journal of General Internal Medicine, 30(8), 11181124. doi:10.1007/s11606-015-3217-6.

18. Wister, A. V., Malloy-Weir, L. J., Rootman, I., \& Desjardins, R. (2010). Lifelong educational practices and resources in enabling health literacy among older adults. Journal of Aging and Health, 22(6), 827-854. doi:10.1177/0898264310373502.

19. Lee, S., \& Choi, S. (2009). Disparities in access to health care among non-citizens in the United States. Health Sociology Review, 18(3), 307-320. doi:10.5172/hesr.2009.18.3.307.

20. Johnson, V. R., Jacobson, K. L., Gazmararian, J. A., \& Blake, S. C. (2010). Does social support help limited-literacy patients with medication adherence?: A mixed methods study of patients in the Pharmacy Intervention for Limited Literacy (PILL) study. Patient Education and Counseling, 79(1), 14-24.

21. Thao, L., \& Yang, Y. (2016). A comparative analysis of Shamanism and Christianity's perspective of mental health in the Hmong community. Retrieved from http://csus-dspace.calstate.edu/bitstream/handle/10211.3/170943/2016Thao\&Lee. pdf? sequence $=3$.

22. Paasche-Orlow, M. K., Parker, R. M., Gazmararian, J. A., Nielsen-Bohlman, L. T., \& Rudd, R. R. (2005). The prevalence of limited health literacy. Journal of general internal medicine, 20(2), 175-184. doi:10.1111/j.1525-1497.2005.40245.x.

23. Sørensen, K., Van den Broucke, S., Fullam, J., Doyle, G., Pelikan, J., Slonska, Z., \& Brand, H. (2012). Health literacy and public health: A systematic review and integration of definitions and models. BMC Public Health, 12(1), 80. doi:10.1186/1471-2458-12-80.

24. Sentell, T., \& Braun, K. L. (2012). Low health literacy, limited English proficiency, and health status in Asians, Latinos, and other racial/ethnic groups in California. Journal of Health Communication, 17(sup3), 82-99. doi:10.1080/10810730.2012.7126 21.

25. Salant, T., \& Lauderdale, D. S. (2003). Measuring culture: a critical review of acculturation and health in Asian immigrant populations. Social Science and Medicine, 57(1), 71-90.

26. Kutner, M., Greenburg, E., Jin, Y., \& Paulsen, C. (2006). The Health Literacy of America's Adults: Results from the 2003 National Assessment of Adult Literacy. NCES 2006-483. National Center for Education Statistics.

27. Martin, L. T., Ruder, T., Escarce, J. J., Ghosh-Dastidar, B., Sherman, D., Elliott, M., Bird, C. E., Fremont, A., \& Lurie, N. (2009). Developing predictive models of health literacy. 
Journal of general internal medicine, 24(11), 1211. doi:10.1007/ s11606-009-1105-7.

28. Wolf, M. S., Gazmararian, J. A., \& Baker, D. W. (2005). Health literacy and functional health status among older adults. Archives of Internal Medicine, 165(17), 1946-1952. doi:10.1001/ archinte.165.17.1946.

29. Koenig, L. B., \& Vaillant, G. E. (2009). A prospective study of church attendance and health over the lifespan. Health Psychology: Official Journal of the Division of Health Psychology, American Psychological Association, 28(1), 117-124. doi:10.1037/a0012984.

30. Krok, D. (2014). The religious meaning system and subjective well-being. Archive For The Psychology Of Religion/Archiv Für Religionspsychologie, 36(2), 253-273. doi:10.1163/15736121-12341288.

31. Krok, D. (2008). The role of spirituality in coping: Examining the relationships between spiritual dimensions and coping styles. Mental Health, Religion and Culture, 11(7), 643-653. doi:10.1080/13674670801930429.

32. Powell, L. H., Shahabi, L., \& Thoresen, C. E. (2003). Religion and spirituality: Linkages to physical health. American Psychologist, 58(1), 36.

33. Lee, S. Y. D., Arozullah, A. M., Cho, Y. I., Crittenden, K., \& Vicencio, D. (2009). Health literacy, social support, and health status among older adults. Educational Gerontology, 35(3), 191-201.

34. Osborn, C. Y., Bains, S. S., \& Egede, L. E. (2010). Health literacy, diabetes self-care, and glycemic control in adults with type 2 diabetes. Diabetes Technology and Therapeutics, 12(11), 913-919. doi:10.1089/dia.2010.0058.

35. Helsel, D. G., Mochel, M., \& Bauer, R. (2004). Shamans in a Hmong American community. Journal of Alternative and Complementary Medicine, 10(6), 933-938. doi:10.1089/ acm.2004.10.933.

36. Kindig, D. A., Panzer, A. M., \& Nielsen-Bohlman, L. (Eds.), (2004). What is health literacy? In Health literacy: A prescription to end confusion. Washington, DC: National Academies Press. http://www.nap.edu/catalog/10883/ health-literacy-a-prescription-to-end-confusion.

37. Pfeifer, M. E., Sullivan, J., Yang, K., \& Yang, W. (2012). Hmong population and demographic trends in the 2010 Census and 2010 American Community Survey. Hmong Studies Journal, 13(2), 1.

38. Vang, C. Y. (2013). In K. Pfeifer, E. Mark, B. Thao (Eds.), The state of the Hmong American community 2013. Washington, DC: Hmong National Development.

39. Baker, D. W. (2006). The meaning and the measure of health literacy. Journal of General Internal Medicine, 21(8), 878-883. 